\title{
(6) OPEN ACCESS \\ Risk of skin and soft tissue infections (including shingles) in patients exposed to anti-tumour necrosis factor therapy: results from the British Society for Rheumatology Biologics Register
}

\author{
James B Galloway, ${ }^{1}$ Louise K Mercer, ${ }^{1}$ Alison Moseley, ${ }^{1,2}$ William G Dixon, ${ }^{1}$ \\ Andrew P Ustianowski, ${ }^{3}$ Matthew Helbert, ${ }^{4}$ Kath D Watson, ${ }^{1}$ Mark Lunt, ${ }^{1}$ \\ Kimme L Hyrich, ${ }^{1}$ Deborah PM Symmons ${ }^{1}$
}

\begin{abstract}
${ }^{1}$ Arthritis Research UK Epidemiology Unit, Manchester Academic Health Sciences Centre, The University of Manchester, Manchester, UK ${ }^{2}$ University of Birmingham, Birmingham, UK ${ }^{3}$ Department of Infectious Diseases, North Manchester General Hospital, Manchester, UK

${ }^{4}$ Department of Immunology, Central Manchester NHS

Foundation Trust,

Manchester, UK
\end{abstract}

\section{Correspondence to \\ Dr Kimme Hyrich, Arthritis Research UK Epidemiology Unit, Manchester Academic Health Sciences Centre, Stopford Building, Oxford Road, The University of Manchester, Manchester, M13 9PT, UK; kimme.hyrich@manchester. ac.uk.}

Accepted 25 March 2012

Published Online First 24 April 2012

\section{ABSTRACT}

Introduction Anti-tumour necrosis factor (TNF) therapy is a mainstay of treatment in rheumatoid arthritis (RA). In 2001, BSRBR was established to evaluate the safety of these agents. This paper addresses the safety of anti-TNF therapy in RA with specific reference to serious skin and soft tissue infections (SSSI) and shingles.

Methods A cohort of anti-TNF-treated patients was recruited alongside a comparator group with active RA treated with non-biological disease-modifying antirheumatic drugs (nbDMARD). 11881 anti-TNF and 3673 nbDMARD patients were analysed. Follow-up was by 6 -monthly questionnaires to patients and clinicians. Analyses considered SSSI and shingles separately. Incidence rates (IR) were calculated and then compared using survival analyses.

Results The crude IR for SSSI were: anti-TNF 1.6/100 patient-years (95\% Cl 1.4 to 1.8); nbDMARD 0.7/100 patient-years (95\% Cl 0.5 to 1.0$)$ and shingles: anti-TNF 1.6/100 patient-years ( $95 \% \mathrm{Cl} 1.3$ to 2.0); nbDMARD $0.8 / 100$ patient-years $(95 \% \mathrm{Cl} 0.6$ to 1.1$)$. Adjusted HR were SSSI 1.4 (95\% Cl 0.9 to 2.4), shingles 1.8 (95\% Cl 1.2 to 2.8). For SSSI, no significant differences were seen between anti-TNF agents. For shingles, the lowest risk was observed for adalimumab (adjusted HR vs nbDMARD) 1.5 (95\% Cl 1.1 to 2.0) and highest for infliximab (HR 2.2; 95\% Cl 1.4 to 3.4)).

Conclusion A significantly increased risk of shingles was observed in the anti-TNF-treated cohort. The risk of SSSI tended towards being greater with anti-TNF treatment but was not statistically significant. As with any observational dataset cause and effect cannot be established with certainty as residual confounding may remain. This finding would support the evaluation of zoster vaccination in this population.

Compared with the general population, skin and soft tissue infections occur around three times more frequently in patients with rheumatoid arthritis (RA). ${ }^{1}$ This is due to a combination of factors, including both the immunosuppressive treatments as well as a complication of the disease itself.

It is now over a decade since the introduction of a new class of biological disease-modifying antirheumatic therapies to the treatment armamentarium for RA: the anti-tumour necrosis factor (TNF) agents. While these treatments have dramatically changed our ability to control the disease, they have also been associated with an increased risk of infection, especially early on after commencing the therapy. ${ }^{2-5}$

In addition, there is evidence suggesting that this risk of infection is not constant across anatomical sites. ${ }^{6}$ Very few data exist specifically examining the risk of skin and soft tissue infections. However, a subgroup of skin infections caused by herpes zoster (shingles), has been studied by other European registries. ${ }^{8}$ Data from a German biologics register (RABBIT) identified an increased risk of shingles in patients treated with monoclonal antibodies against TNF (eg, infliximab, adalimumab but not etanercept, ETNa recombinant TNF receptor fusion protein). ${ }^{9}$

The primary aim of this research was to explore the whether anti-TNF therapy increases the risk of skin and soft tissue infections (including shingles) above that experienced by RA patients treated with more traditional immunosuppressive regimens. The secondary aims were to compare the risks in patients exposed to different anti-TNF agents and to examine whether the risk changed with the duration of exposure.

\section{METHODS}

Ethics approval for this study was obtained from the Multicentre Research Ethics Committee for the northwest of England.

The British Society for Rheumatology Biologics Register (BSRBR) is a prospective observational cohort study. Details of this study including methods have been published previously. ${ }^{10}$ It was established in 2001, and is currently ongoing, with ethical approval to follow patients until at least 2013. It was initiated alongside national recommendations in the UK that all RA patients prescribed anti-TNF therapy should be enrolled on the register. ${ }^{11}$ Only etanercept, infliximab and adalimumab are considered in this study as the other anti-TNF therapies have only recently been introduced into the UK market. Recruitment to the infliximab and etanercept cohorts began from the start of the study, while recruitment to the adalimumab cohort began in 2003. 
A comparison cohort of patients with active RA receiving nonbiological disease-modifying antirheumatic drugs (nbDMARD) was recruited in parallel. Active RA was defined as having a 28 joint count disease activity score (DAS28) $)^{12}$ greater than 4.2. All patients in both cohorts were biological naive at entry. At the time of this analysis, the BSRBR data had over $90 \%$ power to detect a doubling in the rate of both skin infections and shingles in the anti-TNF cohort compared with the comparison cohort.

Patients prescribed biological agents were recruited from across the UK (over 250 hospitals), whereas the comparison cohort was recruited from 29 centres, reflecting a combination of secondary and tertiary care rheumatology centres distributed across the UK.

\section{Baseline assessment}

All patients in this study had a physician diagnosis of RA. Baseline information included demographics, disease duration, a measure of RA disease activity using the DAS28, a measure of self-reported physical function (the health assessment questionnaire; $\mathrm{HAQ},{ }^{13}$ baseline steroid use, smoking history and baseline co-morbidity.

\section{Follow-up}

Follow-up information was collected by postal questionnaire every 6 months from the treating rheumatology team as well as the patients. Patients were also provided with a diary to record details of all hospital attendances and new prescriptions. All patients were flagged with the UK National Health Service Information Centre that reports to the BSRBR on deaths of patients within the study. Adverse events were coded using the Medical Dictionary for Regulatory Activities terminology. All follow-up was censored at 31 December 2009. Follow-up was limited to the first 3 years per patient as information was available from all three sources of reporting (physician, patient and UK National Health Service Information Centre) during this time.

\section{Case definition and verification}

Serious skin and soft tissue infections (SSSI) were analysed separately from shingles. Skin and soft tissue infections were only included if they were serious in nature (defined as resulting in hospitalisation, requiring intravenous antibiotics or causing death). Postoperative infections (ie, any infection occurring within 30 days of surgery) were excluded. An additional proforma requesting further details was sent to the treating rheumatologist in all cases of reported serious infection. As many cases of shingles would not satisfy the definition of serious, all shingles cases were analysed irrespective of the source of reporting or the seriousness of the event. JBG reviewed all soft tissue infections (SSSI) reported to the BSRBR to ensure correct coding. JBG and AM reviewed and additionally categorised shingles cases independently. 'Severe' shingles was defined as being multidermatomal, requiring intravenous antiviral agents, or being a primary reason for hospital admission.

\section{Statistical methods}

Baseline characteristics are presented with $p$ values to assess significant differences between cohorts. Baseline values for the individual anti-TNF cohorts refer to the first biological agent a patient received. For dichotomous variables, a Pearson's $\chi^{2}$ was used. For comparison of continuous variables Wilcoxon rank sum tests were used allowing for non-parametric data. When more than two groups were present the Kruskal-Wallis test was used.
Crude incidence rates were calculated as the number of events per 100 patient-years of follow-up. Patients contributed follow-up in the nbDMARD cohort from the date of registration until their first event, 3 years after registration, 31 December 2009 , last returned follow-up or death, whichever came first. Follow-up was censored at the point of switching for those patients who subsequently started a biological agent. In the antiTNF cohort, patients contributed follow-up time from the first dose of the drug until the first skin infection, 90 days after their first missed dose of anti-TNF agent, 3 years after their first dose, 31 December 2009, last returned follow-up or death, whichever came first. Patients on anti-TNF who switched anti-TNF agents remained under follow-up and contributed follow-up time to their second or subsequent anti-TNF drug. Patients who switched to a non-anti-TNF biological agent had their follow-up censored at the point of switching.

Survival modelling with Cox proportional hazards was used to compare event rates between the groups. An overall HR is presented for the entire follow-up period as well as by 6-month time windows to explore the variation of risk with time. A sensitivity analysis was performed for shingles that compared the rates of serious shingles between the two treatment groups.

Potential confounders were identified before the analysis: age, gender, disease duration, disease severity (DAS28), disability (HAQ score), baseline steroid exposure, smoking status, relevant co-morbidity (diabetes, chronic obstructive pulmonary disease) and year of entry into the study. Adjusting for potential confounders was performed using a propensity model. This involved generating a score for every patient, which reflected the likelihood of them being in the anti-TNF cohort or the nbDMARD cohort based on their baseline information (including all the identified potential confounders). This score was then used as a weight in the survival model. The use of this method in observational studies has been described in detail previously. ${ }^{14}$

Missing baseline data were replaced using multiple imputations with 20 cycles. Patterns of missing follow-up data were compared between cohorts. Different numbers of patients dropped out of follow-up in the nbDMARD and anti-TNF cohorts due to switching therapy (switching from nbDMARD to anti-TNF or other biological agent, or switching from antiTNF to an alternative biological agent). A sensitivity analysis was performed to investigate the extent of bias that may be present due to this differential dropout using a marginal structural model, re-weighting the propensity model every 6 months for the likelihood of dropping out due to switching.

Further details of the statistical methods are available in the supplementary attachment (available online only). All analyses were performed using Stata 10.1.

\section{RESULTS}

\section{Participants}

This analysis included 15554 patients (11881 anti-TNF and 3673 nbDMARD-treated patients). Figure 1 shows the numbers of patients continuing under follow-up at each time point. A breakdown of the reasons for dropping out from follow-up is shown in the supplementary attachment (available online only).

\section{Baseline characteristics}

Anti-TNF-treated patients were younger and a higher percentage was women compared with nbDMARD (table 1). The anti-TNF-treated patients had more severe disease. Differences between patients treated with the three anti-TNF agents were less marked. 
Event rates and hazard estimates comparing nbDMARD with anti-TNF: SSSI

Three hundred and nine SSSI were reported to the registry (269 anti-TNF; 39 nbDMARD). The majority of these were cellulitis $(227 / 309 ; 73 \%)$ with the remainder being coded as abscesses or infected ulcers. Four cases of SSSI were necrotising fasciitis (all in the anti-TNF cohort). Culture information was available for 130/269 (48\%) in the anti-TNF cohort and $15 / 39(38 \%)$ in the nbDMARD cohort. Staphylococci were the most frequently reported organisms in both cohorts (see table 2). There were proportionally more cases of pseudomonal infection in the anti-TNF cohort. The median hospital stay for SSSI was 5.5 days in the anti-TNF-treated

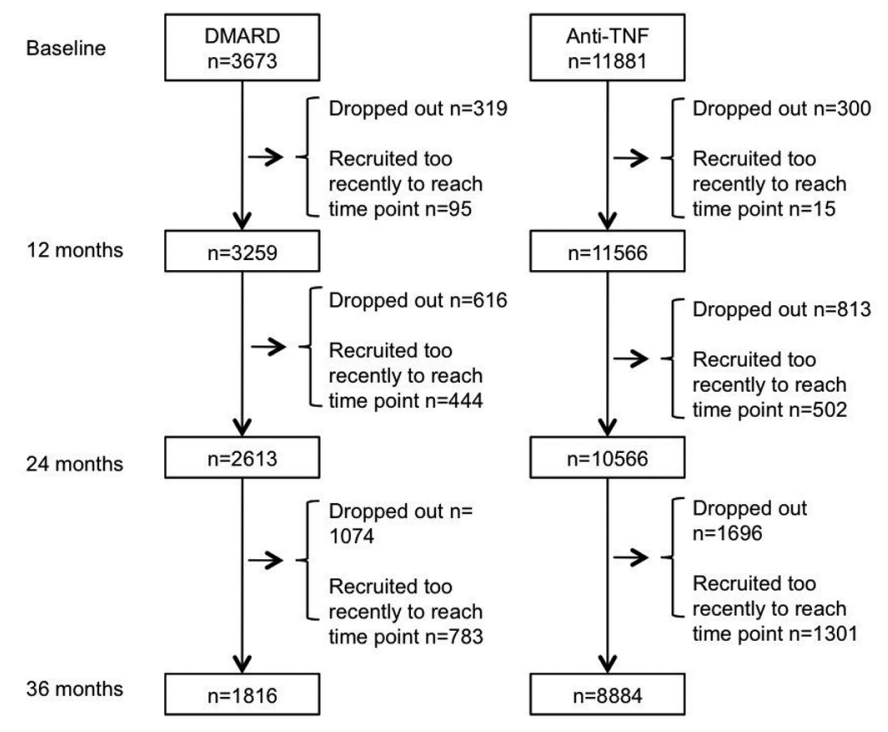

Figure 1 Flowchart showing follow-up of patients. Flowchart showing cumulative numbers of patients in follow-up at each time point. Details of the reasons for dropout are presented in the supplementary data (available online only). DMARD, disease-modifying antirheumatic drug; TNF, tumour necrosis factor. cohort (IOR 3-10) and 5 days in the nbDMARD-treated cohort (IOR 4-15) ( $\mathrm{p}$ value for $\left.\chi^{2}=0.459\right)$. The incidence rate for SSSI was $1.6 / 100$ patient-years in the anti-TNF cohort, compared with $0.7 / 100$ patient-years in the nbDMARD cohort (table 3). The unadjusted HR for SSSI for anti-TNF was 2.2 (95\% CI 1.5 to 3.0$)$.

Univariable adjusted results are available in supplementary table 1 (available online only). The following variables altered the unadjusted result by more than $10 \%$ : age, disability (HAO score) and year of entry into the study. Year of entry into the study reflects the fact that patients recruited earlier in the study had more severe disease (higher DAS28 score, HAQ score, disease duration). The fully adjusted HR using the propensity model for SSSI was 1.3 (95\% CI 0.8 to 2.2 ).

\section{Comparing the individual anti-TNF agents: SSSI}

Using the nbDMARD cohort as the comparison, the lowest risk was seen in the adalimumab cohort (HR 1.1; 95\% CI 0.6 to 2.1). The risks observed for etanercept and infliximab were identical (HR 1.5; 95\% CI 0.9 to 2.5). Compared with etanercept, the HR for SSSI for the monoclonal antibodies combined was not significantly different $(p=0.306)$. The differences did not reach statistical significance when adalimumab was compared with either etanercept or infliximab.

\section{Event rates and hazard estimates comparing nbDMARD with anti-TNF: shingles}

There were 275 cases of shingles in the anti-TNF cohort (incidence $1.6 / 100$ patient-years) and 45 in the nbDMARD cohort (incidence 0.8/100 patient-years; table 4). Twenty-one cases of shingles were classified as severe (anti-TNF 20 (7\%), nbDMARD $1(2 \%)$, p value for $\left.\chi^{2}=0.206\right)$. There were five cases of multidermatomal shingles, and six cases of ophthalmic shingles in the anti-TNF cohort. The one severe case in the nbDMARD cohort was ophthalmic. Univariable adjusted results are available in supplementary table 1 (available online only). The propensity adjusted HR for shingles remained significantly elevated at 1.7 (95\% CI 1.1 to 2.7 ).

Table 1 Baseline characteristics of study population

\begin{tabular}{|c|c|c|c|c|c|c|c|}
\hline Characteristic & $\begin{array}{l}\text { nbDMARD } \\
n=3673\end{array}$ & $\begin{array}{l}\text { All TNF } \\
n=11881\end{array}$ & p Value* & $\begin{array}{l}\text { Etanercept } \\
n=4139\end{array}$ & $\begin{array}{l}\text { Infliximab } \\
n=3475\end{array}$ & $\begin{array}{l}\text { Adalimumab } \\
n=4267\end{array}$ & p Valuet \\
\hline Age, years, mean (SD) & $60(12)$ & $56(12)$ & $<0.001$ & $56(12)$ & $56(12)$ & $57(12)$ & 0.018 \\
\hline Gender, (\% female) & $2652(72)$ & $9053(76)$ & $<0.001$ & $3193(77)$ & $2626(76)$ & $3234(76)$ & 0.203 \\
\hline DAS28,‡ mean (SD) & $5.1(1.3)$ & $6.6(1.0)$ & $<0.001$ & $6.6(1.0)$ & $6.6(1.0)$ & $6.5(1.0)$ & $<0.001$ \\
\hline HAO score,§ mean (SD) & $1.5(0.8)$ & $2.0(0.6)$ & $<0.001$ & $2.1(0.6)$ & $2.1(0.5)$ & $1.9(0.6)$ & $<0.001$ \\
\hline $\begin{array}{l}\text { Disease duration, years, } \\
\text { median (IQR) }\end{array}$ & $6(1,15)$ & $11(6,19)$ & $<0.001$ & $12(6,19)$ & $12(6,19)$ & $10(5,18)$ & $<0.001$ \\
\hline Baseline steroid use, $\mathrm{n}(\%)$ & $845(23)$ & $5228(44)$ & $<0.001$ & $1979(48)$ & $1609(46)$ & 1664 (39) & $<0.001$ \\
\hline Diabetes, n (\%) & $234(6.7)$ & $675(5.8)$ & 0.033 & $255(6)$ & $169(4)$ & $261(6)$ & 0.026 \\
\hline COPD, n (\%) & $304(8)$ & $570(5)$ & $<0.001$ & $222(5)$ & $165(5)$ & $183(4)$ & 0.070 \\
\hline \multicolumn{8}{|l|}{ Smoking, $\mathrm{n}(\%)$} \\
\hline Current & $868(24)$ & $2580(22)$ & 0.001 & $846(21)$ & $757(22)$ & $977(23)$ & 0.029 \\
\hline Ex & $1454(40)$ & $4510(38)$ & & $1576(38)$ & 1314 (38) & $1620(38)$ & \\
\hline Never & $1333(36)$ & 4714 (40) & & 1691 (41) & $1386(40)$ & 1637 (39) & \\
\hline
\end{tabular}

${ }^{*} \mathrm{p}$ Value represents the significance of differences between the nbDMARD and anti-TNF cohorts using $\chi^{2}$ tests for categorical outcomes and Wilcoxon rank sum tests for continuous variables.

tp Value represents the significance of differences between the three anti-TNF drugs using $\chi^{2}$ tests for categorical outcomes and Kruskal-Wallis rank tests for continuous variables. †DAS28 range $0-10 ;>5.1$ high disease activity, 3.2-5.1 moderate activity, 2.1-3.2 low disease activity, $<2.1$ remission.

$\S H A Q$ scale $0-3,3$ indicating worst disability.

COPD, chronic obstructive pulmonary disease; DAS28, 28-joint disease activity score; nbDMARD, non-biological disease-modifying antirheumatic drug; HAQ, health assessment questionnaire; TNF, tumour necrosis factor. 
Comparing the individual anti-TNF agents: shingles

The adalimumab cohort had the lowest risk of shingles compared with nbDMARD (HR 1.5; 95\% CI 0.9 to 2.4) followed by etanercept (HR 1.7; 95\% CI 1.0 to 2.7) and then infliximab (HR 2.2; 95\% CI 1.4 to 3.4). No significant difference was apparent when comparing the rates of shingles with etanercept with the monoclonal antibodies combined. However, within the monoclonal antibody class, the risk of shingles was significantly higher with infliximab when compared with adalimumab (HR 1.5; 95\% CI 1.1 to 2.0). Excluding patients who switched anti-TNF treatment during follow-up did not alter these findings.

\section{Event rate over time: SSSI and shingles}

The hazard for both SSSI and shingles varied over time, with a higher risk noted early on in therapy (figure 2). An early peak in hazard was more marked for SSSI, in which in the first 6 months of therapy 71 events were reported in the anti-TNF cohort (IR 5.0/100 patient-years; $95 \%$ CI 3.9 to 6.4) compared with three in the nbDMARD cohort (IR 0.9/100 patient-years; $95 \%$ CI 0.2 to 2.6), resulting in an adjusted HR of 3.5 (95\% CI 0.6 to 19.2). Review of the cases occurring within this early time period did not reveal any unusual patterns. The type of infection was predominantly cellulitis.

\section{Differential dropout during follow-up}

A significantly greater proportion of the nbDMARD cohort dropped out from follow-up because of switching to biological therapy $(12.9 \%$ vs $0.7 \%)$. The patients who switch inevitably

Table 2 Organisms responsible for skin and soft tissue infections

\begin{tabular}{lcc}
\hline Organism & Anti-TNF, n & DMARD, n \\
\hline Gram-positive species & & \\
Staphylococcus aureus & & \\
Coagulase negative staphylococci & 8 & 11 \\
Streptococcus spp. ${ }^{*}$ & 11 & 1 \\
Gram-negative species & & 1 \\
Pseudomonas aeruginosa & 20 & 1 \\
Proteus mirabilis & 2 & 1 \\
Stenotrophomonas maltophilia & 1 & 0 \\
Escherichia coli & 1 & 0 \\
Klebsiella spp. $t$ & 1 & 0 \\
Morganella morganii & 1 & 0 \\
Enterobacter spp. & 1 & 0 \\
No culture information available & 140 & 24
\end{tabular}

*Staphylococci were reported to be methicillin-resistant species in $24(29 \%)$ of the antiTNF episodes and four (36\%) of the DMARD cases.

tReports in which subspecies information was not provided are listed by generic 'spp.' The species breakdown for soft tissue infections shows that staphylococcus was the most prevalent organism in both groups. Pseudomonas was observed more frequently in the anti-TNF cohort.

DMARD, disease-modifying antirheumatic drug; TNF, tumour necrosis factor. comprise a cohort with higher disease activity. As disease severity and the associated requirement for corticosteroid therapy are independent risk factors for infections, this will result in an effective depletion of susceptible individuals from the nbDMARD cohort. The HR for SSSI for anti-TNF adjusting for dropout using the marginal structural model was $0.9(95 \%$ CI 0.5 to 1.4). For shingles, the hazard was influenced less by the adjustment for dropout (HR 1.5; 95\% CI 1.0 to 2.4).

\section{DISCUSSION}

To date, only limited information on the site-specific risk of infection with anti-TNF therapy has been available. This paper examined skin infections in detail and demonstrated a nonsignificant increase in SSSI, and a significantly increased risk of shingles.

The absolute risk of SSSI in the anti-TNF cohort was more than double that seen in the nbDMARD cohort. This finding should encourage clinicians to be vigilant for skin infections in patients starting anti-TNF therapy. It is likely that some of the additional risk of SSSI seen in the anti-TNF cohort is attributable to other risk factors. This is demonstrated both by the reduction in the HR from 2.2 to 1.4 after adjustment for confounders and the disappearance of any association after adjusting for differential dropout.

There was a higher proportion of pseudomonal infections in the anti-TNF cohort. While the clinical significance of skin culture results is not always clearcut (some of the reported pathogens could represent commensals) the difference is an interesting observation and suggests that TNF inhibition may modify the risk of SSSI. With this in mind, it is important to consider that while the reported HR for SSSI with anti-TNF was not statistically significant at 1.4 , the upper limit of the $95 \%$ CI was 2.4 . Therefore, it is important to acknowledge that a clinically relevant increased risk has not been excluded.

The increased risk of shingles with anti-TNF is important because shingles is associated with substantial morbidity. ${ }^{15}$ In BSRBR the incidence of shingles in the anti-TNF cohort was almost $2 \%$ per year. Adjusting for confounders or differential dropout made only a minor difference in the HR estimates, suggesting that the association between anti-TNF and shingles is less likely to reflect selection bias. The estimated number needed to treat for 1 year with anti-TNF to see one additional case of shingles was 128. In the USA, the Centers for Disease Control and Prevention (CDC) has recommended that all adults over the age of 60 years be offered vaccination against shingles (Zostavax) irrespective of previous exposure status. ${ }^{16}$ The vaccine is a live therapy and should be used with caution in people who are immunosuppressed. The CDC recommends that, while low dose corticosteroids or methotrexate are not contraindications, the vaccine should not be administered to individuals already established on anti-TNF therapy. These findings should

Table 3 Incidence and HR of SSSI

\begin{tabular}{|c|c|c|c|c|c|}
\hline Result & $\begin{array}{l}\text { nbDMARD } \\
\mathrm{n}=3673\end{array}$ & $\begin{array}{l}\text { All anti-TNF } \\
\mathrm{n}=11881\end{array}$ & $\begin{array}{l}\text { Etanercept } \\
\mathrm{n}=4139\end{array}$ & $\begin{array}{l}\text { Infliximab } \\
n=3475\end{array}$ & $\begin{array}{l}\text { Adalimumab } \\
n=4267\end{array}$ \\
\hline Follow-up (pyrs) & 5416 & 17048 & 6122 & 4529 & 6397 \\
\hline SSSI events & 39 & 269 & 120 & 79 & 67 \\
\hline $\begin{array}{l}\text { SSSI incidence (/100 } \\
\text { patient-years) }\end{array}$ & $0.7(0.5-1.0)$ & $1.6(1.4-1.8)$ & $2.0(1.6-2.3)$ & $1.7(1.4-2.2)$ & $1.0(0.8-1.3)$ \\
\hline SSSI unadjusted HR & Ref & $2.1(1.5-3.0)$ & $2.8(1.9-4.0)$ & $2.5(1.7-3.7)$ & $1.5(1.0-2.2)$ \\
\hline SSSI adjusted $\mathrm{HR}^{*}$ & Ref & $1.3(0.8-2.2)$ & $.5(0.9-2.5)$ & $1.5(0.9-2.5)$ & $1.1(0.6-2.1)$ \\
\hline
\end{tabular}

${ }^{*}$ Adjusted rates using propensity modelling described in the Methods section and using multiple imputations to replace missing baseline variables.

nbDMARD, non-biological disease-modifying antirheumatic drug; SSSI serious skin and soft tissue infections; TNF, tumour necrosis factor. 
Table 4 Incidence and HR of shingles

\begin{tabular}{|c|c|c|c|c|c|}
\hline Result & $\begin{array}{l}\text { nbDMARD } \\
\mathrm{n}=3673\end{array}$ & $\begin{array}{l}\text { All TNF } \\
\mathrm{n}=11881\end{array}$ & $\begin{array}{l}\text { Etanercept } \\
\mathrm{n}=4139\end{array}$ & $\begin{array}{l}\text { Infliximab } \\
\mathrm{n}=3475\end{array}$ & $\begin{array}{l}\text { Adalimumab } \\
n=4267\end{array}$ \\
\hline Follow-up (patient-years) & 5417 & 17048 & 6122 & 4529 & 6397 \\
\hline Shingles events & 45 & 275 & 99 & 91 & 85 \\
\hline $\begin{array}{l}\text { Shingles incidence } \\
\text { (/100 patient-years) }\end{array}$ & $0.8(0.6-1.1)$ & $1.6(1.4-1.8)$ & $1.6(1.3-2.0)$ & $2.0(1.6-2.5)$ & $1.3(1.1-1.6)$ \\
\hline Shingles unadjusted HR & Ref & $1.9(1.4-2.6)$ & $1.7(1.2-2.5)$ & $2.4(1.7-3.4)$ & $1.7(1.2-2.5)$ \\
\hline Shingles adjusted $\mathrm{HR}^{*}$ & Ref & $1.7(1.1-2.7)$ & $1.7(1.0-2.7)$ & $2.2(1.4-3.4)$ & $1.5(0.9-2.4)$ \\
\hline
\end{tabular}

${ }^{*}$ Adjusted rates using propensity modelling described in the Methods section and using multiple imputations to replace missing baseline variables. nbDMARD, non-biological disease-modifying antirheumatic drug; TNF, tumour necrosis factor.

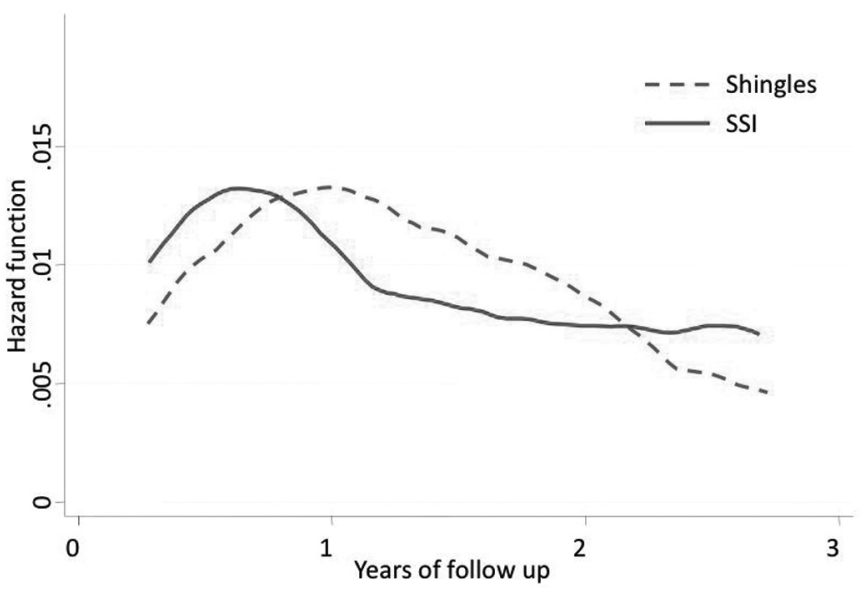

Figure 2 Hazard for soft tissue infections (SSI) and shingles over time in anti-tumour necrosis factor (TNF)-treated cohort. Demonstration of the time-varying risk of events in the anti-TNF cohort. Event rates were higher earlier on during treatment in both cohorts.

encourage clinicians to consider vaccination before exposure to anti-TNF therapy. It is worth noting that current estimates of any vaccine uptake among RA patients are low. ${ }^{17}$ Further study into the utility of vaccination in RA cohorts is clearly warranted.

A hypothesis that etanercept carried a lower risk of shingles compared with the monoclonal antibodies (infliximab and adalimumab) arose from research published by the German biologics registry. ${ }^{9}$ In this study we observed an increased risk of shingles with all three drugs. The hazard estimates from the BSRBR lie within the CI limits reported by the German registry, and the finding of a significant association for all drugs may simply reflect the greater power of the BSRBR dataset.

The risk of SSSI and shingles was greatest early on after initiating anti-TNF therapy. This pattern has been noted previously and a number of explanations have been hypothesised. ${ }^{18}$ It is likely that there are a number of factors contributing to the pattern. Undoubtedly, a 'healthy user' effect is present, but in addition, there is also likely to be changes in other time-varying confounders such as steroid exposure.

The strengths of this study are the large sample size combined with a simultaneously recruited comparator cohort allowing for robust comparisons of rare outcomes that could not be studied in the context of clinical trials. The multiple reporting methods incorporating both information from patients and clinicians further strengthens the validity of the results.

There are also limitations to acknowledge. Although these data allow us to say that patients chosen to start on anti-TNF therapy within the BSRBR are at higher risk of shingles and SSSI than the comparator cohort, we cannot say with certainty that this increased risk is directly attributable to the anti-TNF treatment. There remains the possibility that residual confounding exists.
For example, the lack of high quality information about steroid use during follow-up precluded the analysis of steroids as a timevarying covariate. Diagnostic suspicion bias may also have played a role as clinicians may have had a lower threshold for admitting patients on anti-TNF therapy. However, if this was the case we might have expected to see shorter hospital admission durations in the anti-TNF arm, and this was not the case. Conversely, we may have underestimated the risk of SSSI or shingles with anti-TNF as it is possible that clinicians were less likely to prescribe anti-TNF therapy to patients who were perceived to have the highest risk of infection. Also, other potential unmeasured confounders such as frailty are not captured in our baseline covariates.

The study population reflects a predominantly Caucasian cohort based in the UK, and so generalisations to cohorts of other ethnicities or in regions where the background rates of infection differ may not be appropriate. Although the nbDMARD-treated patients were recruited from a limited number of centres, both cohorts came from a mixture of teaching and non-teaching hospitals spread geographically across the UK. While the observational design has important limitations, the data presented here reflect real world experience of anti-TNF therapy in RA in the UK, without patients having to fit the strict selection criteria often applied by randomised controlled trials.

\section{CONCLUSIONS}

There is an increased incidence of skin infections (including shingles) in RA patients prescribed anti-TNF therapy. Some of this risk may be a reflection of other co-morbidities or the concurrent use of corticosteroids. A better understanding of the individual risk profiles of different biological agents will enable patients and clinicians to make more personalised decisions regarding care.

Acknowledgements The authors acknowledge the enthusiastic collaboration of all consultant rheumatologists and their specialist nurses in the UK in providing the data. In addition, the authors acknowledge the support from Dr lan Griffiths (past) and Professor David Isenberg (current), chairs of the BSRBR Management Committee; Professor Gabriel Panayi, Professor David GI Scott, Dr Andrew Bamji, Professor Deborah Bax and Professor DL Scott, presidents of the British Society for Rheumatology (BSR) during the period of data collection, for their active role in enabling the register to undertake its tasks, and to Samantha Peters (CEO of the BSR), Mervyn Hogg, Nia Taylor and members of the BSRBR Scientific Steering Committee. The authors also acknowledge the seminal role of the BSR Clinical Affairs Committee for establishing national biological guidelines and recommendations for such a register. Finally, the authors would like to acknowledge the substantial contribution of Andy Tracey, Katie McGrother and Dr Mark Lunt in database design and manipulation and Professor Alan Silman in his previous role as a principal investigator of the BSRBR and BSRBR Control Centre Consortium. The BSRBR Control Centre Consortium consists of the following institutions (all in the UK): Antrim Area Hospital, Antrim (Dr Nicola Maiden); Cannock Chase Hospital, Cannock Chase (Dr Tom Price); Christchurch Hospital, Christchurch (Dr Neil Hopkinson); Royal Derby Hospital, Derby (Dr Sheila O'Reilly); Dewsbury and District Hospital, Dewsbury (Dr Lesley Hordon); Freeman Hospital, Newcastle-upon-Tyne (Dr lan Griffiths); Gartnavel General Hospital, Glasgow (Dr Duncan Porter); Glasgow Royal Infirmary, Glasgow (Professor Hilary Capell); Haywood Hospital, Stoke-on-Trent (Dr Andy Hassell); Hope Hospital, Salford (Dr Romela Benitha); King's College Hospital, London (Dr Ernest Choy); Kings Mill Centre, Sutton-In-Ashfield (Dr David Walsh); 
Leeds General Infirmary, Leeds (Professor Paul Emery); Macclesfield District General Hospital, Macclesfield (Dr Susan Knight); Manchester Royal Infirmary, Manchester (Professsor lan Bruce); Musgrave Park Hospital, Belfast (Dr Allister Taggart); Norfolk and Norwich University Hospital, Norwich (Professor David Scott); Poole General Hospital, Poole (Dr Paul Thompson); Queen Alexandra Hospital, Portsmouth (Dr Fiona McCrae); Royal Glamorgan Hospital, Glamorgan (Dr Rhian Goodfellow); Russells Hall Hospital, Dudley (Professor George Kitas); Selly Oak Hospital, Selly Oak (Dr Ronald Jubb); St Helens Hospital, St Helens (Dr Rikki Abernethy); Weston General Hospital, Weston-super-Mare (Dr Shane Clarke/Dr Sandra Green); Withington Hospital, Manchester (Dr Paul Sanders); Withybush General Hospital, Haverfordwest (Dr Amanda Coulson); North Manchester General Hospital (Dr Bev Harrison); Royal Lancaster Infirmary (Dr Marwan Bukhari) and The Royal Oldham Hospital (Dr Peter Klimiuk).

Contributors $\mathrm{JBG}$ and $\mathrm{LKM}$ were involved in all aspects of this research. $\mathrm{KLH}$ and DPMS are both principal investigators for the BSRBR and oversaw this research. AM worked on case verification and data analysis. WGD, MH and APU advised on the analysis and contributed to writing the manuscript. ML provided statistical support. KDW contributed to data collection, analysis and editing the manuscript. The BSRBR control centre consortium was involved in the recruitment of patients to the control arm.

Funding Funding for this project was provided by the British Society for Rheumatology.

Competing interests The BSR commissioned the Biologics Register (BSRBR) as a UK-wide national project to investigate the safety of biological agents in routine medical practice. DPMS and KLH are principal investigators on the BSRBR. BSR receives restricted income from UK pharmaceutical companies, presently Abbott Laboratories, Biovitrum, Merk Sharpe \& Dohme, Pfizer and Roche. This income finances a wholly separate contract between the BSR and the University of Manchester. The principal investigators and their team have full academic freedom and are able to work independently of pharmaceutical industry influence. All decisions concerning analyses, interpretation and publication are made autonomously of any industrial contribution. Members of the Manchester team, BSR trustees, committee members and staff complete an annual declaration in relation to conflicts of interest. The authors declare no other competing interests.

Ethics approval Ethics approval for this study was obtained from the Multicentre Research Ethics Committee for the northwest of England.

Provenance and peer review Not commissioned; externally peer reviewed.

Open Access This is an Open Access article distributed in accordance with the Creative Commons Attribution Non Commercial (CC BY-NC 3.0) license, which permits others to distribute, remix, adapt, build upon this work non-commercially, and license their derivative works on different terms, provided the original work is properly cited and the use is non-commercial. See: http://creativecommons.org/ licenses/by-nc/3.0/

\section{REFERENCES}

1. Doran MF, Crowson CS, Pond GR, et al. Frequency of infection in patients with rheumatoid arthritis compared with controls: a population-based study. Arthritis Rheum 2002;46:2287-93.
2. Galloway JB, Hyrich KL, Mercer LK, et al. Anti-TNF therapy is associated with an increased risk of serious infections in patients with rheumatoid arthritis especially in the first 6 months of treatment: updated results from the British Society for Rheumatology Biologics Register with special emphasis on risks in the elderly. Rheumatology (Oxford) 2011;50:124-31.

3. Askling J, Fored CM, Brandt L, et al. Time-dependent increase in risk of hospitalisation with infection among Swedish RA patients treated with TNF antagonists. Ann Rheum Dis 2007:66:1339-44.

4. Listing J, Strangfeld A, Kary S, et al. Infections in patients with rheumatoid arthritis treated with biologic agents. Arthritis Rheum 2005;52:3403-12.

5. Curtis JR, Patkar N, Xie A, et al. Risk of serious bacterial infections among rheumatoid arthritis patients exposed to tumor necrosis factor alpha antagonists. Arthritis Rheum 2007;56:1125-33.

6. Dixon WG, Watson K, Lunt M, et al. Rates of serious infection, including site-specific and bacterial intracellular infection, in rheumatoid arthritis patients receiving antitumor necrosis factor therapy: results from the British Society for Rheumatology Biologics Register. Arthritis Rheum 2006;54:2368-76.

7. García-Doval I, Pérez-Zafrilla B, Descalzo MA, et al. Incidence and risk of hospitalisation due to shingles and chickenpox in patients with rheumatic diseases treated with TNF antagonists. Ann Rheum Dis 2010;69:1751-5.

8. Salmon-Ceron D, Tubach F, Lortholary 0, et al. Drug-specific risk of non-tuberculosis opportunistic infections in patients receiving anti-TNF therapy reported to the 3-year prospective French RATIO registry. Ann Rheum Dis 2011;70:616-23.

9. Strangfeld A, Listing J, Herzer P, et al. Risk of herpes zoster in patients with rheumatoid arthritis treated with anti-TNF-alpha agents. JAMA 2009;301: 737-44.

10. Hyrich KL, Watson KD, Isenberg DA, et al. The British Society for Rheumatology Biologics Register: 6 years on. Rheumatology (Oxford) 2008;47:1441-3.

11. National Institute for Health and Clinical Excellence. Guidance on the use of etanercept and infliximab for rheumatoid arthritis. London, UK: NICE, 2002.

12. Prevoo ML, van't Hof MA, Kuper HH, et al. Modified disease activity scores that include twenty-eight-joint counts. Development and validation in a prospective longitudinal study of patients with rheumatoid arthritis. Arthritis Rheum 1995;38: 44-8.

13. Kirwan JR, Reeback JS. Stanford Health Assessment Questionnaire modified to assess disability in British patients with rheumatoid arthritis. Br J Rheumatol 1986;25:206-9.

14. Curtis LH, Hammill BG, Eisenstein EL, et al. Using inverse probability-weighted estimators in comparative effectiveness analyses with observational databases. Med Care 2007;45(10 Suppl 2):S103-7.

15. Oxman MN, Levin MJ, Johnson GR, et al. A vaccine to prevent herpes zoster and postherpetic neuralgia in older adults. N Engl J Med 2005;352:2271-84.

16. Harpaz R, Ortega-Sanchez I, Seward J. Prevention of herpes zoster. MMWR Morb Mortal Wkly Rep 2008;50:1-30.

17. Zhang J, Delzell E, Xie F, et al. The use, safety, and effectiveness of herpes zoster vaccination in individuals with inflammatory and autoimmune diseases: a longitudinal observational study. Arthritis Res Ther 2011;13:R174.

18. Askling J, Dixon W. The safety of anti-tumour necrosis factor therapy in rheumatoid arthritis. Curr Opin Rheumatol 2008;20:138-44. 